\title{
EL ENFOQUE COGNITIVO COMO ALTERNATIVA AL ESTUDIO INMANENTE DEL SIGNIFICADO: EL CASO DE LA ESCUELA DE SEMÁNTICA DE LA LAGUNA*
}

\author{
Francisco Javier García Yanes \\ IES Mencey Bencomo, Tenerife
}

\section{Resumen}

Tradicionalmente, se ha considerado que el principio de inmanencia constituye la piedra fundacional y condición sine qua non de la lingüística moderna. Su aplicación al estudio del significado, sin embargo, conlleva múltiples dificultades, tanto teóricas como prácticas, que ponen en cuestión su viabilidad. Sobre la base del realismo corporeizado (Lakoff y Johnson 1999), la lingüística cognitiva replantea las bases filosóficas del modelo y ofrece una alternativa al estructuralismo que recupera los vínculos entre el lenguaje, el pensamiento y la realidad externa. Una vez constatada la existencia de importantes afinidades entre esta nueva corriente y el estructuralismo europeo, y en especial con respecto a los trabajos de la Escuela de Semántica de La Laguna, como corriente heterodoxa dentro del estructuralismo, se sugiere aquí la asunción por parte de esta última de los presupuestos filosóficos y principios metodológicos del enfoque cognitivo del estudio del lenguaje, como medio para la superación de las dificultades que hoy en día afronta su modelo.

Palabras Clave: significado, lingüística cognitiva, inmanentismo, estructuralismo, perspectivismo inmanente.

\author{
COGNITIVE LINGUISTICS AS AN ALTERNATIVE TO THE IMMANENT APPROACH
}

TO THE STUDY OF LANGUAGE: THE CASE OF LA LAGUNA SCHOOL OF SEMANTICS

\section{Abstract}

The immanent approach to language has traditionally been seen as both the foundation stone and a necessary requirement for modern linguistics. However, when applied to the study of meaning, it faces both theoretical and practical difficulties, which cast doubt on its validity. As an alternative, cognitive linguistics builds on embodied realism (Lakoff and Johnson 1999) to challenge the philosophical foundations of the model, and restore the links between language, thought and reality. Relevant similarities, it is posed here, can be found between cognitive linguistics and European structuralism, especially in the case of the La Laguna School of Semantics (LLSS), as the heterodox branch of structuralism it is. On this basis, it might be worth for the LLSS to adopt cognitive linguistics' philosophical foundations and methodological principles as a way to overcome the difficulties its model currently faces.

KEYWORDS: meaning, cognitive linguistics, immanentism, structuralism, immanent perspectivism. 


\section{INTRODUCCIÓN}

Pese a que Saussure nunca hiciese referencia explícita al concepto de inmanencia en sus escritos (Zinna 2014: 26; Arrivé 2014: 57), se le suele atribuir el mérito de haber establecido las bases para el estudio inmanente y autónomo del lenguaje, $y$, con ello, para el desarrollo de la lingüística moderna. El proyecto, formulado por Hjelmslev (1980 [1943]), y asumido por el estructuralismo, persigue definir las unidades de una lengua «con independencia de la realidad», en función de las relaciones opositivas en las que, según esta perspectiva, se sustenta su sistematización (García Padrón 1997: 90; vid. también Zinna 2015: 337-338). En lo que respecta al estudio del significado, tal objetivo implica, según se entiende, la delimitación precisa del contenido de las unidades lingüísticas con exclusión de los aspectos conceptuales vinculados a ellas.

La historia de la aplicación de tal programa al ámbito semántico es, sin embargo, la de un proyecto en gran parte frustrado. De hecho, no parece siquiera que los autores que aún hoy en día son referencia obligada dentro del marco de la semántica estructuralista hayan sido capaces, como trataré de mostrar a continuación, de ofrecer ejemplos de análisis coherentes con los principios postulados.

Los desarrollos aportados por la Escuela de Semántica de La Laguna (ESLL) suponen, en este sentido, un intento extremo de llevar a término el proyecto estructuralista del estudio inmanente del lenguaje en el ámbito del significado. Sin embargo, el mismo inmanentismo que, durante estos años, ha servido de base para la ingente labor llevada a cabo por la ESLL es, según argumentaré, el responsable de las principales limitaciones que presenta el modelo propuesto por esta corriente heterodoxa del estructuralismo. Como vía alternativa para la resolución de dichas limitaciones, y tras la constatación de un conjunto importante de afinidades entre la ESLL y la lingüística cognitiva -mayores incluso que las concomitancias existentes entre esta y el estructuralismo ortodoxo-, se sugiere aquí la conveniencia de la asunción por parte de la escuela lagunera de los fundamentos filosóficos y principios metodológicos cognitivistas, como instrumento de validación de las propuestas hechas hasta ahora, y medio para superar los obstáculos señalados.

Con tal intención, se procederá en el presente artículo, en primer lugar, a analizar las limitaciones que presenta la aplicación de los principios del inmanentismo al estudio del significado lingüístico, tal como este ha sido abordado por sus principales adalides (apartado 2). A continuación, se expondrán los rasgos característicos del enfoque particular -dentro del marco inmanentista- adoptado por la ESLL, se valorarán sus principales aportaciones y se señalarán sus mayores inconvenientes (apartado 3). En el apartado 4 se dibujarán las líneas maestras de la lingüística cognitiva, especialmente en lo que respecta a sus fundamentos filosóficos y

* El autor desea agradecer los valiosos comentarios y sugerencias recibidos de los evaluadores anónimos de la Revista de Filología de la Universidad de La Laguna, que sin duda han contribuido a mejorar la versión inicial del presente trabajo. La responsabilidad sobre el contenido del texto es, en todo caso, exclusiva del autor. 
metodológicos, en contraste con el enfoque inmanente. Los apartados 5 y 6 se dedicarán a la exposición de los puntos de contacto que justifican la propuesta -aquí formulada- de adopción de tales fundamentos filosóficos y principios metodológicos por parte de la ESLL: el apartado 5 mostrará las afinidades existentes entre la lingüística cognitiva y el estructuralismo europeo, mientras que el apartado 6 señalará las concomitancias -bien heredadas del estructuralismo o bien específicas- que la ESLL presenta con respecto al enfoque cognitivo. Por último, en el apartado 7, se extraerán las conclusiones pertinentes.

\section{LOS PILARES DEL PROYECTO INMANENTE}

Saussure -como más tarde, siguiendo sus enseñanzas, también el estructuralismo- concibe el significado de las unidades lingüísticas como "valor», esto es, como fenómeno de carácter exclusivamente estructural, definido «no positivamente por su contenido, sino negativamente por sus relaciones con los otros términos del sistema» (1991 [1916]: 147). El inconveniente de esta definición -como señala Dick M. Bakker- es que desemboca en un razonamiento circular:

In a minimal system, consisting of two elements, one element, say, $\mathrm{A}$, is different from an element that is not A, say, Z. Should one ask what that implies, the answer has to be: A differs from $Z$, which in its turn is characterized by being different from $A$. Then the circle is round, and it does not matter, of course, how many elements there are between A and Z (cit. en Elffers 2012: 27)'.

Para romper esta circularidad, no parece quedar otra solución que recurrir a lo conceptual como fuente de la que extraer los ingredientes que permitan definir el significado de una unidad lingüística en particular. Esto es lo que, aparentemente, hace el propio Saussure. Ciertamente, el maestro ginebrino plantea que «todos los valores están siempre constituidos: $1 .^{\circ}$, por una cosa desemejante susceptible de ser trocada por otra cuyo valor está por determinar; $2 .^{\circ}$, por cosas similares que se pueden comparar con aquella cuyo valor está por ver» (Saussure 1991 [1916]: 144-145). $\mathrm{Al}$ mismo tiempo, afirma que en la lengua «solo hay diferencias sin términos positivos» (Saussure 1991 [1916]: 151). Se deduce, por tanto, que deberá ser algún elemento "extralingüístico» el que aporte las semejanzas imprescindibles -según se plantea- para establecer el valor en cuestión. Tal elemento parece aportarlo, en la concepción de Saussure, un nivel de contenido conceptual de carácter no lingüístico (Taylor 1999: 41; Elffers 2012: 26).

Pese a lo que se afirma explícitamente en el Curso de lingüistica general, Saussure concibe este nivel conceptual no como una masa amorfa en la que resulta

${ }^{1}$ El planteamiento de Saussure resulta, de hecho, paradójico, dada la imposibilidad práctica de establecer el sistema -tal como este es concebido en el Curso de lingüística general- sin organizar primero, según su contenido, los elementos mismos que lo constituyen. 
imposible -a menos que se cuente con la ayuda de los signos lingüísticos- distinguir dos ideas (1991 [1916]: 140), sino como un continuo de dos dimensiones -de ahí el concepto de "vecindad» (Saussure 1991 [1916]: 145)-, en el que las ideas se hallan yuxtapuestas según su grado de similitud. Dada esta realidad, la labor de las unidades lingüísticas sería la de marcar sobre dicha superficie las divisiones que señalan los límites entre unos signos y otros, sin que quede espacio alguno sin cubrir (vid. García Padrón 1997: 54-55).

Desde esta perspectiva, se entiende por qué Saussure concibe la significación como formada no solo por el valor, sino también, más allá de su concepción de lo estrictamente lingüístico, por «la propiedad que tiene la palabra de representar una idea» (Saussure 1991 [1916]: 143), propiedad que podríamos parafrasear como la capacidad para remitir a un concepto. Es, efectivamente, la materia conceptual de estas «ideas» -no parece haber alternativa- la que aporta el ingrediente necesario para alcanzar la importante conclusión de que dos ideas son «vecinas», esto es, para sentar la base de la comparación sobre la que luego se pueda establecer -ya solo por oposición- el valor del signo lingüístico. E, igualmente, es a esta materia a la que Saussure recurre para explicar de qué depende el valor específico de dos unidades «vecinas», como pueden ser las palabras inglesas «mutton» $\mathrm{y}$ «sheep»:

El español carnero o el francés mouton pueden tener la misma significación que el inglés sheep, pero no el mismo valor, y eso [...] porque al hablar de una porción de comida ya cocinada y servida a la mesa, el inglés dice mutton y no sheep (Saussure, 1991 [1916]: 145).

El nacimiento de la glosemática, a mediados del siglo xx, iba a suponer la acentuación del inmanentismo apuntado por Saussure (vid. Arrivé 2014: 49-50; Paolucci 2014), al plantear la concepción del signo lingüístico -como señala García Padrón- como función solidaria de una forma de contenido con una forma de expresión, independientemente de sus respectivas sustancias (sentido y sonido), y llevar a cabo con ello una nítida distinción, en la descripción semántica de las lenguas, entre «sus vertientes sistemática (forma del contenido) e interpretativa (sustancia del contenido)" (1997: 91). En sus Prolegómenos a una teoría del lenguaje, Hjelmslev (1980 [1943]: 75-80) insiste, además, tal como había hecho ya previamente Saussure, en el carácter amorfo del pensamiento previo a su conformación por parte de las distintas lenguas.

Con todo, y pese a que su objetivo es el de dotar a la lingüística de un mecanismo meramente formal que le permita "perseguir una constancia que no se apoye en ninguna "realidad" exterior al lenguaje» (Hjelmslev 1980 [1943]: 18), lo cierto es que, en la práctica, tampoco el padre de la glosemática nos ofrece en su obra ejemplos concretos de análisis del significado de los signos léxicos como entidades caracterizadas meramente por oposición con respecto a otras unidades, sin sustancia alguna. Muy al contrario, sus análisis presuponen una concepción del significado como entidad conceptual definida positivamente -por afinidad con otras unidades- y de carácter composicional, en la que una forma de contenido de una unidad puede funcionar como elemento constitutivo de otras, según analogías extraídas de la sustancia del contenido. Así se constata cuando afirma, por ejemplo, que, en el 
procedimiento de inventariado «puramente mecánico» de las unidades del plano del contenido, se podrá prescindir de elementos como «man», «woman», «boy» o "girl», puesto que tales entidades pueden, a su vez, descomponerse en «human being», "he» $\mathrm{y}$ «she» (Hjelmslev 1980 [1943]: 101).

El nacimiento de la semántica estructural, años más tarde, no iba a suponer tampoco una novedad con respecto a los planteamientos desde los que hasta entonces se había propuesto abordar el estudio del significado: una vez más, Coseriu (1986 [1977]: 132-133) insistirá en la necesidad de una clara delimitación entre lo lingüístico y lo conceptual, advirtiéndonos contra la identificación de significado y clase. Y, pese a ello, la definición de las unidades «lingüísticas» seguirá descansando, en parte, en la sustancia del contenido, y nunca solo en principios opositivos funcionales puros, como requería el modelo (Trujillo 1988; Taylor 1999: 29)². Tal hecho resulta patente en la propia definición que da Coseriu del "campo léxico», como «estructura paradigmática constituida por unidades léxicas que se reparten una zona de significación común y que se encuentran en oposición inmediata las unas a las otras» (1986 [1977]: 170; el subrayado es mío), retomando la idea del continuo conceptual de dos dimensiones como punto de partida para el establecimiento del valor lingüístico ${ }^{3}$.

Por otra parte, como señala Lyons, la mayoría de los semantistas estructurales, incluidos Hjelmslev y Coseriu, suscriben alguna versión del análisis componencial, un enfoque fundado «en la tesis de que el sentido de todo lexema puede analizarse por medio de un conjunto de componentes de sentido (o rasgos semánticos) más generales, algunos de los cuales serán comunes a distintos lexemas del vocabulario" (1980: 299). Tales rasgos, tal cual son concebidos por esta corriente, se obtienen una vez más, como apunta Kleiber (1995 [1990]: 26-27), del sustrato conceptual cuya existencia previa al lenguaje era negada por Saussure.

\section{LA ESCUELA DE SEMÁNTICA DE LA LAGUNA}

La Escuela de Semántica de La Laguna nace a finales de los años sesenta, a iniciativa del profesor Gregorio Salvador, y de la mano de la lexemática, dentro del marco de la investigación semántica estructuralista más ortodoxa (Coseriu 1986 [1977]; García Padrón 1997: 68-75). Pronto, sin embargo, Ramón Trujillo, uno de sus miembros más destacados, tomaría distancia con respecto a sus orígenes, asumiendo desde planteamientos afines a la glosemática la necesidad de un estudio estrictamente inmanente del significado (Batista Rodríguez 1997; García Padrón 1997). Daba comienzo así a una profunda labor de reflexión e investigación, que habría

${ }^{2}$ Los propios fundadores de la semántica estructural se dirigieron en alguna ocasión reproches en este sentido: es el caso de Coseriu, quien considera que la propuesta de Pottier está basada en el análisis de «dominios objetivos de la realidad extralingüística» (1986 [1977]: 171).

3 Obsérvese incluso el mantenimiento de la metáfora geográfica, evidente en el uso del sustantivo «zona». 
de desembocar finalmente en el cuestionamiento de la utilidad para la lingüística de la noción de "campo léxico" o "semántico", en tanto que estructura-entiende Trujillo (1988: 91-101) - meramente conceptual, delimitada sobre la base de semejanzas de naturaleza cultural (cfr. García Padrón 1990).

En su lugar, la ESLL planteará el estudio semántico desde el enfoque denominado por Batista Rodríguez (1997: 28) perspectivismo inmanente, basado en una concepción del significado como realidad de naturaleza intuitiva o sensitiva, inconsciente e inefable (Trujillo 1988; Morera 2007: 100), como "“orientación” que marca, dentro de unos límites semánticos precisos, las pautas de los infinitos sentidos contextuales posibles» (Trujillo 1988: 82). Cada significado lingüístico es, para esta corriente, un objeto «único» y "concreto», en el sentido de entidad real y primaria, anterior a la interpretación; y no "un plano secundario o abstracto", ni «la síntesis de alguna clase de objetos físicos o mentales» $\mathrm{o}$ «un conjunto de inferencias deducidas de [las] realidades significadas» (Trujillo 1988: 6, 10) ${ }^{4}$. Es en este sentido en el que Trujillo insiste en que el significado de un término no es un concepto (1988: 91). Desde esta nueva perspectiva, la ESLL presenta las unidades lingüísticas como entidades "autosemánticas», susceptibles de ser explicadas con independencia de la realidad (García Padrón 1997: 90).

Como se ha señalado, las aportaciones de la ESLL suponen un intento extremo de llevar a término el proyecto estructuralista del estudio semántico del lenguaje desde una perspectiva inmanente. No obstante, el carácter sumamente esquemático de los significados lingüísticos postulados por esta escuela, convertidos en un mero molde conformador del referente -sea este cual sea-, marca, sin duda, una clara distancia con respecto al estructuralismo. Así, los análisis estructuralistas ortodoxos, al construir sus campos semánticos a partir del continuo conceptual de las «significaciones» de Saussure, de claro carácter taxonómico, tienen como techo el nivel de la denotación, esto es, el de las acepciones ligadas a ámbitos específicos del conocimiento de la realidad. Tal límite se mantiene -dentro de la corriente estructuralista-incluso tras el surgimiento de la pragmática, en la medida en que la nueva subdisciplina lingüística propone una división neta entre un significado literal o descontextualizado («lo que se dice»), o denotación primaria; y un significado intencional ("lo que se quiere decir»), entendido como interpretación, o significado contextualizado en la situación comunicativa (vid. García Padrón 1997: 174).

Para la ESLL, en cambio, ni las unidades lingüísticas ni sus combinaciones remiten por defecto, como pretendía el estructuralismo, a una denotación primaria, ligada a un ámbito específico del conocimiento de la realidad, y motivo de su adscripción a uno u otro campo semántico; sino que, muy al contrario, están vinculadas directamente a un "potencial semántico», presente en todos los niveles de análisis, «sin modificarse en su esencia íntima» y, a la vez, variando «en las múlti-

${ }^{4}$ Aclara, no obstante, Trujillo (1988: 11) que tales afirmaciones no deben entenderse desde las perspectivas filogenética u ontogenética, sino únicamente en relación con cómo se presentan las unidades lingüísticas en la mente del hablante en el momento de su uso, en unas circunstancias concretas. 
ples circunstancias contextuales y de habla en que coincide con otros signos» (García Padrón 1989: 6). El uso contextualizado de los signos lingüísticos no debe, pues, sustituir a un sentido literal previo, dado que el significado solo se vincula - de forma convencional o individual- a una determinada denotación y designación en el discurso, y no dentro del propio sistema (García Padrón 1989).

Partiendo de esta nueva concepción del significado, Trujillo (1988: 99, 103) llega incluso a defender la independencia semántica de los significados léxicos, renunciando con ello al carácter estructural de buena parte de las unidades lingüísticas. La posibilidad de que «el significado tenga la estructura del modelo lógico de un análisis componencial» es igualmente descartada por este autor, con el argumento de que «la descomposición en rasgos implica siempre la consideración del significado como concepto, y no como percepción» (Trujillo 1988: 55).

Tales planteamientos, sin embargo, tomados de manera estricta, parecen conducir al estudio inmanente del significado a un callejón sin salida a la hora de enfrentarse, en la práctica, al análisis de las unidades lingüísticas. Por ello, otros autores de esta misma corriente buscan una aproximación al significado más cercana a los principios del estructuralismo, que -aunque manteniendo su independencia con respecto a la conceptualización de ámbitos específicos de la realidad- admita tanto la existencia de relaciones de oposición inmediata entre las unidades del plano del contenido (Morera 2000a: 8) como la idea de que tales unidades se puedan descomponer en «un grupito más o menos amplio, jerarquizado y cerrado de rasgos semánticos no sígnicos (es decir, sin significante propio) universales", que -siguiendo a Hjelmslev (1980 [1943]: 71) - son denominados figuras de contenido (Morera 2007: 107).

\subsection{Valoración de las aportaciones de la Escuela de Semántica de La Laguna}

Resulta evidente el interés de la labor de revisión crítica de los postulados del estructuralismo llevada a cabo por la ESLL, así como el valor de sus reflexiones teóricas acerca de la naturaleza del significado, o de sus aportaciones al estudio de las unidades que constituyen la lengua española. Con todo, un reconocimiento como el aquí expresado no debe impedir apreciar igualmente los inconvenientes que presentan las propuestas de esta escuela.

En primer lugar, el marco teórico general dibujado por la ESLL suscita algunas interrogantes de difícil solución, propias del enfoque rigurosamente inmanentista asumido por esta corriente. Así, la enorme resistencia a la influencia del contexto gramatical y denotativo, y, en definitiva, al cambio, que se les atribuye a los significados lingüísticos (vid. Morera 2007: 236) plantea el problema de la explicación de su origen y adquisición. Ciertamente, Morera (2007: 236) no niega la posibilidad del cambio semántico; sin embargo, tal posibilidad parece desbordar el poder explicativo del modelo, de modo que, a la hora de señalar la razón última del fenómeno, el autor recurre a la idea de lo «asistemático» o lo «fortuito» (Morera 2007: 236-237). De forma paralela, el alto grado de desconexión que se postula para los significados lingüísticos con respecto a sus variantes denotativas y designativas dificulta enormemente, desde el punto de vista ontogenético, la explica- 
ción de cómo tales significados pueden ser aprendidos a partir de actos comunicativos concretos 5 .

Tampoco es posible obviar el hecho de que Morera, debido a sus principios estructuralistas, se ve obligado a confeccionar extensas clasificaciones con el fin de justificar los significados de las unidades lingüísticas en función de oposiciones binarias. De su aplicación al análisis de unidades concretas resultan a menudo descripciones extremadamente complejas, que ponen en tela de juicio la viabilidad psicológica del modelo, así como su utilidad para el estudio del comportamiento de las unidades lingüísticas en la realidad del discurso ${ }^{6}$.

Por otro lado, su método adolece de cierto grado de subjetivismo, así como de una excesiva confianza en el recurso a la introspección y la intuición del propio analista, rasgos estos que cuestionan la validez científica de las hipótesis propuestas, en la medida en que imposibilitan en gran medida su falsación por parte de otros investigadores ${ }^{7}$.

Por último, al haber desechado el concepto de campo semántico, la única estructura "verdaderamente idiomática» (Morera 2007: 155) que le resta a la ESLL para el estudio del significado de las unidades léxicas (raíces) no es otra que la familia de palabras. No deja de suponer, sin embargo, un serio inconveniente para esta teoría la constatación, expresada por el propio Morera, de que, desde la perspectiva adoptada, «resulta a veces absolutamente imposible determinar la significación invariante de raíces con poco o nulo desarrollo gramatical y denotativo" (2007: 217).

\section{EN BUSCA DE UNA ALTERNATIVA}

Las dificultades que afronta el enfoque inmanente a la hora de abordar el estudio del significado han supuesto que el proyecto estructuralista, como se reconoce a menudo (vid. Trujillo 1988; Osuna García 2005, entre otros), no haya lle-

5 Por ejemplo, el significado «desplazamiento de algo que es interno al sujeto, porque lo produce, posee, domina, etc.-en proceso-situado en una relación de movimiento de alejamiento respecto de un punto de referencia-puntualmente acabado-como abstracción no esencial activa», que corresponde, según se propone, a la palabra delator (Morera 2007: 66), se concretaría en una denotación tan aparentemente distante de tal significado como la recogida por el DRAE (2014) como única acepción: «denunciante, acusador».

${ }^{6}$ Véase, por ejemplo, la descripción del significado del sufijo -uno como «cuantificación externa-desde el exterior-continua-limitada-sin apego al límite-extensa-marcada-no definida» (Morera 2005: 219); o la de la palabra «naturaleza» como «acción de nacer-puntualmente acabada (nato)-como abstracción no esencial pasiva (natura)-externamente expandida en todas las direcciones de su materia semántica (natural)-como emanación pasiva (naturaleza)» (2005: 47).

7 Dicho subjetivismo se observa, por ejemplo, cuando afirma que lo que caracteriza a los sufijos -io y -ez es el hecho de que la «cuantificación externa desde el interior [...] que implica [...] sacar materia semántica del elemento nuclear» se produce en este caso "por emanación», en la medida en que «la materia semántica que se saca se presenta como potencia, fuerza o creación de la materia semántica del elemento nuclear» (Morera 2005: 53). 
gado a producir los resultados esperados. Con todo, algunos autores insisten aún hoy en día en la necesidad de mantener el objetivo de la descripción inmanente del significado lingüístico, aunque para ello deban renunciar a algunos de los elementos centrales del estructuralismo ortodoxo. Así, mientras que la ESLL opta por desprenderse del concepto de campo semántico, Osuna García, por su parte, propone sustituir la idea de significado como concepto por la del significado como función, entendida esta como el «uso que hacemos de los signos y construcciones» (2005: 81).

La lingüística cognitiva toma justamente la vía opuesta: por un lado, defiende que el significado no puede ser sino una realidad de naturaleza conceptual; y, por otro, reniega del inmanentismo, al entender que el valor de las unidades lingüísticas no depende, en última instancia, de relaciones internas al sistema, sino, muy al contrario, del vínculo que tales unidades mantienen (por medio de la esquematización) con respecto a escenas relevantes, desde la perspectiva de la experiencia corporeizada del ser humano.

\subsection{LA LINGÜÍSTICA COGNITIVA Y SUS FUNDAMENTOS FILOSÓFICOS}

La lingüística cognitiva, surgida en los años setenta, se caracteriza por presentar el lenguaje como capacidad integrada en la cognición general, y defender, en consecuencia, la necesidad de atender a los principios teóricos y avances aportados por la psicología cognitiva como vía para el desarrollo de un estudio sistemático y científico del lenguaje (Evans 2014).

En cuanto a la cuestión que aquí nos ocupa, bien podría considerarse que el elemento central que separa a esta corriente de las pertenecientes al paradigma formal (vid. Figueroa 1994: 17-25) es precisamente el que tiene que ver con la viabilidad misma del proyecto planteado por el inmanentismo. Y esto es así porque la lingüística cognitiva -aun cuando desde sus propias filas se haya reivindicado a menudo su raiga mbre saussureana (vid. Elffers 2012: 20) - se asienta de hecho sobre unos fundamentos filosóficos sustancialmente distintos a los del estructuralismo.

Ciertamente, las escuelas surgidas a partir de las ideas de Saussure parecen construir su concepción del lenguaje sobre una visión dualista y racionalista (vid. Hessen 1970 [1926]; Lakoff y Johnson 1999; Johnson y Lakoff 2002), según la cual la realidad externa es un puro caos que solo adquiere sentido una vez sometida a la conformación que de ella hace el lenguaje. En consecuencia, el estructuralismo concibe la cognición humana como un fenómeno de una sola instancia -el sistema lingüístico-, ajena a la realidad externa; con olvido absoluto de la dimensión corporal del ser humano y de la función vital de la percepción.

La ESLL, por su parte, amplía la visión racionalista del estructuralismo recuperando para su concepción de la cognición humana una forma de realismo natural, al modo aristotélico (vid. Hessen 1970 [1926]: 38-39), según la cual tanto los conceptos como las propiedades percibidas provienen de las cosas, independientemente de la conciencia cognoscente (vid. Trujillo 88: 91). Esta recuperación, sin embargo, se lleva a cabo dentro de esta corriente con el único fin de subrayar la dife- 
rencia entre el ámbito conceptual y el lingüístico, que sigue conservando intacto su carácter inmanente.

La ciencia cognitiva, por el contrario, niega la posibilidad de que exista un nivel de representación mental del todo extraño a la realidad externa, y reconstruye, sobre la base de los presupuestos filosóficos del experiencialismo o realismo corporeizado (Lakoff y Johnson 1986 [1980]; 1999), los vínculos entre ambas dimensiones, recordándonos que nuestra mente no es, en último término, sino un dispositivo de supervivencia (Lakoff y Johnson 1999: 91; Evans y Green 2006: 48) surgido de la interacción del ser humano -a través de la percepción ${ }^{8}$ - con esa realidad que el estructuralismo consideraba tan ajena al pensamiento y el lenguaje?

\subsection{SignificAdo y CONCEPTO}

Una vez rotos los lazos con la realidad, el estructuralismo, en su afán de explicar el fenómeno lingüístico, construye un sistema cerrado, que se define y justifica a sí mismo en un espacio abstracto y atemporal (Bondron 2014: 22; Arrivé 2014: 53 y ss.), absolutamente extraño -al menos en teoría- a la experiencia real (tanto interna como externa) a la que da sentido. El inmanentismo es, pues, para el estructuralismo una necesidad: dada la ausencia de vínculo alguno entre el lenguaje y la realidad externa, el contenido de los significados lingüísticos no puede provenir de otra fuente que no sean las propias relaciones internas que se establecen entre tales significados. Las dificultades que el modelo afronta a la hora de justificar tanto el origen histórico y la transformación de las unidades de una lengua como el proceso por el cual estas son aprendidas por cada uno de sus hablantes son, pues, previsibles, en tanto que dadas por los propios presupuestos filosóficos de los que se parte.

La psicología y la lingüística cognitiva, por el contrario, son capaces de explicar la naturaleza y el origen -filogenético y ontogenético (vid. Tomasello 2013 [2008]) - de las unidades que conforman una lengua a partir de su propia concepción del fenómeno lingüístico, rescatando así el estudio científico de esta facultad humana del limbo de inmanencia y atemporalidad en el que el estructuralismo - primero- y el generativismo - tras él- lo habían sumergido. Por muy abstractas o esquemáticas que sean, las unidades del lenguaje, según las concibe la lingüística cognitiva, tienen un significado que no depende (únicamente) de las relaciones internas que se establecen entre ellas, sino que se adquiere y desarrolla a través de la

${ }^{8}$ En sentido amplio, esto es, incluyendo la interocepción (percepciones del propio cuerpo: movimiento, consciencia de la postura corporal, etc.) y la introspección (estados mentales, sentimientos, etc.) (Barsalou 1999).

9 Por supuesto, no se pretende aquí atribuir a la lingüística cognitiva el mérito de liderar - mucho menos de monopolizar- la reacción contra el enfoque inmanente dentro del ámbito del estudio del lenguaje. Tal inquietud, de hecho, constituye uno de los rasgos definitorios del paradigma funcional (Figueroa 1994: 17-25), al cual suele adscribirse la propia lingüística cognitiva (Langacker 2007: 422; Nuyts 2007). 
interacción del individuo con la realidad externa. Los significados lingüísticos no son, pues, ni pueden ser otra cosa que conceptos -esto es, productos de la cognición humana-, caracterizados no por presentar un origen distinto al de otros conceptos, sino, en todo caso, por su elevado nivel de convencionalización y esquematización (Evans 2009; 2015).

Así, se entiende que el hablante construye el sistema lingüístico de manera gradual, a partir de determinadas asociaciones de formas (o estructuras fonológicas) y significados, realizadas en sus diversas experiencias comunicativas (Evans 2016: 288-289). Los elementos que constituyen las unidades lingüísticas están ya presentes en sus «instancias», de modo que la abstracción de las unidades a partir de su uso se consigue de forma directa, producto de la mera repetición y del consecuente refuerzo selectivo y convencionalización de patrones recurrentes (Langacker 2009: 628). Tal concepción implica el carácter dinámico de las unidades del lenguaje, sujetas siempre a su reformulación en función de los ejemplos concretos de uso presentes en las distintas situaciones comunicativas en las que participe el individuo a lo largo de su vida (Kemmer y Barlow 2000). El proceso, además, es circular: pese a tener su origen en el uso, las unidades lingüísticas, una vez adquirido un cierto grado de convencionalización y esquematización, tienden a ser utilizadas como patrones o modelos, frente a otras unidades menos consolidadas (Evans 2014: 289).

Esta definición del significado como concepto no supone necesariamente, como asume Trujillo (1988), su subordinación a la realidad externa, ni la renuncia a una concepción del lenguaje como fuerza creadora de realidades. De hecho, la lingüística cognitiva propone tal definición, como hemos visto, sin aceptar ninguna de las dos consecuencias previstas por el lingüista lagunero. Esto es posible porque, en primer lugar, el realismo corporeizado rechaza la idea - propia del realismo natural- de que los conceptos sean el resultado de una actividad intelectual consciente posterior a la experiencia; y sostiene, en cambio, que el proceso de formación espontánea y uso de conceptos constituye, en sí mismo, lo que entendemos como «realidad». Además, la teoría filosófica en la que se apoya la lingüística cognitiva niega tajantemente que los conceptos puedan definirse a partir de las características de la realidad objetiva; muy al contrario, entiende que los mecanismos cognitivos desempeñan un papel fundamental en el proceso de categorización, hasta el punto de que la mayoría de los conceptos adquirieren al menos parte de su estructura interna por medio de la adopción de la estructura de otros conceptos, por la vía de la proyección metafórica de un dominio conceptual sobre otro (Lakoff y Johnson 1999: 18-19, cap. 18; Gibbs 1996).

\subsection{VALORACión DE LA LiNGüística COGNITIVA}

Como todo constructo teórico, tanto la lingüística cognitiva como el realismo corporeizado presentan puntos sujetos a discusión o susceptibles de ser revisados. En cuanto a los fundamentos filosóficos de la lingüística cognitiva, puede resultar polémico el intento de naturalización de la filosofía (vid. Zahavi 2009) que representa el realismo corporeizado, en la medida en que hace depender el valor de 
las teorías filosóficas de su compatibilidad con los métodos y resultados de la ciencia cognitiva. El estudio de la mente y el lenguaje, defienden Lakoff y Johnson (1999: 78-79), no puede partir de presupuestos apriorísticos que -tal como ocurre en el caso del inmanentismo- determinen la naturaleza tanto de los datos seleccionados como de los resultados obtenidos. Con el fin de minimizar esta posibilidad, la lingüística cognitiva incluye entre sus principios metodológicos el conocido como compromiso cognitivo, según el cual los modelos propuestos deben resultar coherentes con evidencias provenientes de la mayor cantidad de metodologías y de fuentes tan diversas como sea posible, además de compatibles con lo que se sabe acerca de la mente, tanto desde el punto de vista cognitivo como neuronal (Lakoff 1990: 40; Evans 2011$)^{10}$. En este sentido, el valor del realismo corporeizado, como fundamento filosófico de la lingüística cognitiva, no está en su parsimonia, sino en su capacidad -sostienen Johnson y Lakoff (2002) - para dar cuenta de las evidencias empíricas ${ }^{11}$.

Más allá de este aspecto, a la propuesta de Lakoff y Johnson (1999) se le ha reprochado su excesiva simplificación de la tradición filosófica occidental (Haser 2005), así como el carácter individualista de su concepción de la mente y del lenguaje, con olvido de sus dimensiones sociales, culturales y normativas (Rakova 2002; Zlatev 2007; 2009). A este respecto, Dirven y Ruiz de Mendoza Ibáñez (2010: 35-38) plantean la necesidad de una contextualización del realismo corporeizado dentro del marco de la fenomenología -en la línea de los trabajos de Geeraerts (1985; 1993)-, que reporte a la teoría una mayor solidez filosófica en lo que atañe a aspectos como la unión e interacción entre el cuerpo y la mente, la conexión entre la percepción externa e interna, la naturaleza de la conciencia y la intencionalidad, o las dimensiones sociales y culturales de la «corporeización».

En lo que a las aportaciones concretas de la lingüística cognitiva se refiere, no se puede obviar tampoco la evidente falta de consenso que se da a día de hoy dentro de esta corriente con respecto a cuestiones trascendentales para la disciplina, como la definición de los conceptos de esquema de imagen (vid. Zlatev 2005; 2009) o prototipo (vid. sección 6), el posible carácter componencial del significado (vid. Evans y Green 2006: 61-63) o la necesidad o no de una delimitación clara entre lo lingüístico y lo conceptual (vid. Evans 2009; 2015: 265-269), entre otras.

Aun así, Dirven y Ruiz de Mendoza Ibáñez (2010: 38) no dejan de destacar la posición privilegiada de la lingüística cognitiva -en comparación con otras corrientes del estudio del lenguaje- desde el punto de vista epistemológico, tanto por la flexibilidad de su marco general como por su vinculación a los métodos y los avances de la psicología cognitiva experimental.

${ }_{10}$ Dentro del marco del funcionalismo lingüístico, Simon S. Dik, en sintonía con este principio de la lingüística cognitiva, propone también como requisito para los estudios lingüísticos el que sus propuestas no resulten incompatibles con los resultados de las investigaciones llevadas a cabo dentro del ámbito de la psicología (vid. Ruiz de Mendoza Ibáńez y Galera Masegosa 2014: 23-25).

11 Tanto Gibbs (2005) como Bergen (2012) ofrecen una amplia exposición de la base empírica que sustenta el realismo corporeizado. 


\section{PUNTOS DE CONTACTO ENTRE EL ESTRUCTURALISMO EUROPEO Y LA LINGÜÍSTICA COGNITIVA}

Pese a las diferencias existentes entre los fundamentos filosóficos que las sustentan -anteriormente expuestas-, el estructuralismo europeo y la lingüística cognitiva comparten una serie de principios básicos que permiten establecer vías de comunicación entre ambas corrientes.

En primer lugar, tanto el estructuralismo europeo como la lingüística cognitiva defienden la importancia del significado como elemento indispensable del estudio del lenguaje (vid. Coseriu 1986 [1973]: 119; Langacker 1987: 12). Con ello se distancian claramente de la tradición lingüística estadounidense, reacia, desde los trabajos de Bloomfield, a la inclusión del significado en el análisis lingüístico (vid. García Padrón 1997: 42-44).

Del mismo modo, ambas corrientes coinciden en su consideración de lo semántico como realidad mental - «entidad psíquica» o «fenómeno cognitivo»-, distinta por tanto del estado de cosas de la realidad; y en el consiguiente rechazo de la idea del lenguaje como simple nomenclatura (vid. Saussure 1991 [1916]: 87, 143; Coseriu 1969 [1967]: 11-12; Valenzuela et al. 2016 [2012]: 42-43). Tal concepción marca una diferencia radical con respecto a otras perspectivas del estudio del lenguaje, tales como la semántica formal (o lógica de las condiciones de verdad) o la semántica generativista, en las que el significado es concebido desde un enfoque plenamente referencial, esto es, como correspondencia con el estado de cosas que se da en el mundo (o mundos posibles).

Pese a sus afinidades con el paradigma funcional (Soto 2001; Nuyts 2007), la lingüística cognitiva entiende, además, en sintonía con el estructuralismo europeo (Saussure 1991 [1916]; Hjelmslev 1980 [1943]), que todo texto está construido sobre una serie de unidades mínimas, los signos o símbolos lingüísticos (Langacker 2007: 422; 2008: 30), que sirven de punto de partida y anclaje de los procesos textuales de construcción del significado (Harder 2009; Evans 2009).

Asimismo, al parecer de ambas corrientes, los signos lingüísticos sobre los que están construidos los textos, y que constituyen las distintas lenguas, consisten en asociaciones de una unidad de expresión con una unidad de contenido (Saussure 1991 [1916]: 89, 135-153; Hjelmslev 1980 [1943]: 75; Langacker 1987: 11).

El enfoque cognitivo y el estructuralismo europeo coinciden de nuevo a la hora de señalar que los signos lingüísticos, en la realidad de los textos concretos, están sometidos a diversos grados de variación y convencionalización (Coseriu 1986 [1977]: 187-202; Taylor 2003: 160-161). Ambas corrientes se distancian, sin embargo, en lo que respecta a la naturaleza del vínculo que, en último término, mantiene la unidad del signo lingüístico, en tanto que el estructuralismo únicamente admite como tal la existencia de una invariante de contenido, mientras que el enfoque cognitivista suele decantarse, en su lugar, por el estudio de la motivación entre los diversos sentidos de una unidad, haciendo uso, para ello, de herramientas teóricas como la metonimia, la metáfora y la teoría del prototipo (vid. Lakoff 1987; Taylor 2003). Aun así, es posible encontrar dentro del marco de la lingüística cognitiva autores que proponen, en sintonía con el estructuralismo, la existencia de sig- 
nificados monosémicos altamente esquemáticos (vid. Langacker 2008: 100, 117); o que admiten la posibilidad de fórmulas mixtas (vid. Taylor 1999; 2003: 160-161). Por otro lado, las dificultades afrontadas a la hora de delimitar los distintos sentidos de una unidad (vid. Evans y Green 2006: 339-352) han llevado a algunos autores a reconocer que quizás la lingüística cognitiva haya pecado en sus inicios de una reacción excesiva contra el concepto de monosemia, y a abogar, en consecuencia, por una revalorización de la función del contexto en la conformación de los diversos sentidos de las unidades lingüísticas (Evans y Green 2006: 352-355; Dirven y Ruiz de Mendoza Ibáñez 2010: 46-52).

\subsection{FALSAS INCOMPATIBILIDADES: VALOR, ESTRUCTURA Y PROTOTIPO}

A la cuestión relativa al nivel de esquematización y generalidad de los significados lingüísticos, que se acaba de señalar, es necesario añadir otras que tradicionalmente han sido tenidas por diferencias irreconciliables entre ambas corrientes, y que, sin embargo, no son tales.

Así, habrá que insistir en que la idea que la lingüística cognitiva tiene del significado no se opone necesariamente a las nociones estructuralistas de valor y estructura, definidas en función de relaciones internas de oposición (vid. Taylor 1999: 30-31). De hecho, ambos conceptos figuran en los modelos propuestos por investigadores representativos de este movimiento, tales como Radden y Dirven (2007: 19-20) o Langacker (2008: 263) ${ }^{12}$.

Tampoco la teoría del prototipo, contra la que han arremetido figuras relevantes del estructuralismo (vid. Coseriu 1990), constituye una diferencia irreconciliable entre ambas posturas, en la medida en que sus diversas formulaciones no han estado exentas de discusión dentro de la propia corriente cognitiva (vid. Geeraerts 2016 [1989]; Kleiber 1995 [1990]; Komatsu 1992; Cifuentes Honrubia 1992; Wierzbicka 1996; Goldstone et al. 2017: 304, entre otros). Efectivamente, la propia Rosch, a los pocos años de haber presentado su propuesta, ya advertía contra el error de reificar la noción de prototipo: según la autora, el prototipo no debía entenderse como un modelo de representación de las categorías conceptuales, sino únicamente como una restricción sobre los modelos propuestos, que podrían adoptar diversos formatos, siempre y cuando fuesen capaces de explicar la existencia de los efectos de prototipicidad detectados (Rosch 1999 [1978]: 36-41). La reducción del concepto de prototipo a meros «efectos prototípicos» (Rosch 1999 [1978]; Lakoff 1987), sumada al carácter estático y amodal de la teoría (vid. Fauconnier 1999: 615;

12 No obstante, el papel desempeñado por este tipo de relaciones opositivas dentro de los modelos propuestos por estas dos corrientes es sustancialmente distinto: mientras que tales relaciones son, por principio, imprescindibles para el estructuralismo; para la lingüística cognitiva, por el contrario, tienen un carácter secundario, subordinado, en todo caso, a la vinculación que mantienen los significados lingüísticos con respecto a escenas relevantes desde la perspectiva de la experiencia corporeizada del ser humano. 
Barsalou 1999: 587), y a su dependencia con respecto a factores estadísticos, ciegos a la naturaleza del significado que se está analizando (Goldstone et al. 2017: 291 y ss.), parece, de hecho, haber allanado el camino al cuestionamiento de la validez del modelo como teoría del concepto y la categorización, hasta el punto de que algunos autores (Evans y Green 2006: 249) consideran que, a día de hoy, la teoría del prototipo ha sido superada en muchos aspectos por otras más recientes, como la de sistemas de símbolos perceptuales (Barsalou 1999).

En cuanto a la aplicación del concepto de prototipo al ámbito de la variación del signo lingüístico, la tendencia mayoritaria dentro de la lingüística cognitiva sigue siendo, efectivamente, la de insistir en la búsqueda de un prototipo central que sirva de referencia a los diversos sentidos de una unidad. No hay, sin embargo, acuerdo entre los investigadores sobre el sentido que, en cada caso, debe ser considerado como prototípico, ni tampoco sobre los criterios para el establecimiento de tal sentido central (Gries 2006). En último término, parece claro que, de aceptarse su relevancia para el estudio del significado lingüístico, el concepto de prototipo -entendido como consecuencia de las relaciones establecidas entre los distintos sentidos de una misma unidad lingüística (Lakoff 1987) - no resulta tampoco incompatible con una concepción del significado como la defendida por el estructuralismo, marcada por la presencia de una serie de rasgos o atributos necesarios y suficientes, tal como demuestran los trabajos de Wierzbicka (1996: cap. 4).

\section{AFINIDADES ENTRE LA ESLL Y LA LINGÜÍSTICA COGNITIVA}

Las relaciones que se han establecido en el apartado anterior entre la lingüística cognitiva y el estructuralismo europeo son compartidas igualmente por la ESLL, en tanto que esta no es sino un desarrollo -bajo la fuerte influencia de la glosemática de Hjelmslev (1980 [1943]) y de la lexemática de Coseriu (1986 [1977])de las ideas centrales planteadas por Saussure (1991 [1916]).

De este modo, encontramos que la ESLL coincide con el enfoque cognitivo y la corriente estructuralista europea en defender -con más énfasis aún, si cabe- la importancia del significado como elemento indispensable del estudio del lenguaje (Trujillo 1988: 29).

Igualmente esencial resulta para el modelo de la ESLL la consideración de lo semántico como realidad mental, así como la disociación que tal planteamiento supone entre la dimensión semántica de un texto y la realidad externa. De hecho, tanto la ESLL como la lingüística cognitiva se caracterizan por subrayar la posibilidad que ofrece el lenguaje de, aprovechando esta disociación, someter una misma situación objetiva a un número infinito de conceptualizaciones distintas, por medio de productos lingüísticos igualmente diversos (Morera 2000a: 11, 16; Langacker 1987: 487-488; Boogaart y Reuneker 2017).

La ESLL comparte, además, con el enfoque cognitivo el principio de que las lenguas son, ante todo, mecanismos semánticos que proporcionan posibilidades expresivas, como «intuiciones» o "plantillas» que modelan la percepción de la rea- 
lidad, así como la construcción e interpretación de un texto (Trujillo 1988; Langacker 1987: 5; cap. 3).

Ambas corrientes afirman, asimismo, que las lenguas están constituidas únicamente por signos o símbolos lingüísticos, de naturaleza bien léxica o bien sintáctica o relacional (Morera 2000b: 7-14; Evans 2016: 286-287), ninguno de los cuales carece de significado propio (Morera 2000a: 16; Langacker 2008: 24-26) ${ }^{13}$. De este modo, tanto la ESLL como el enfoque cognitivo se alejan claramente de la concepción que escuelas como el generativismo tienen de las lenguas como estructuras formadas por un conjunto de unidades léxicas y una serie de reglas de combinación (sintaxis).

En cuanto a la naturaleza del signo lingüístico, tanto la corriente cognitiva como la ESLL llevan la concepción que de él tiene Saussure -como asociación de una unidad de expresión con una unidad de contenido- hasta su último extremo, hasta el punto de que ambas corrientes coinciden, si bien por caminos distintos, en negar la posibilidad de sinonimia dentro de una lengua (Trujillo 1988: 16-17, 95; Evans 2009: 209-210).

Por último, no falta tampoco en la bibliografía de la ESLL la referencia a la idea, fundamental para la lingüística cognitiva, de que los diversos sentidos de una unidad están unidos por medio de relaciones semánticas (metáfora, metonimia, especificación y generalización) (Morera 2007: 105, 160, 198; Lakoff 1987).

\subsection{Aproximaciones específicas de la ESLL con Respecto al ENFOQue Cog- NITIVO}

A este conjunto numeroso de afinidades -heredadas del estructuralismo europeo- que comparten la ESLL y la lingüística cognitiva habría que añadir aún otras similitudes que tienen su origen en el carácter idiosincrático de las propuestas de la Escuela. De hecho, podría afirmarse que el distanciamiento llevado a cabo por la ESLL en las últimas décadas con respecto de la ortodoxia estructuralista ha sido paralelo a la adopción, quizás no del todo consciente, de elementos propios de los enfoques de tipo cognitivista.

Así, en lo que respecta a la consideración de su objeto de estudio, la ESLL se acerca a la lingüística cognitiva al incluir entre sus objetivos -si bien tímidamentela atención a la dimensión psicológica del lenguaje. Ciertamente, los autores de la ESLL no se limitan en sus publicaciones a plantear el modelo propuesto desde una perspectiva formal, como un constructo meramente abstracto y teórico, cuya vali-

13 Dentro del ámbito de la lingüística cognitiva, la idea del signo lingüístico como construcción -esto es, como asociación estable de forma y significado o función, independientemente de su grado de complejidad y nivel de descripción (desde el morfológico al discursivo) - constituye el elemento definitorio de las conocidas como gramáticas de construcciones, entre las cuales se incluye no solo la gramática de construcciones cognitiva, de Goldberg (2006), sino también la gramática cognitiva de Langacker (vid. Evans y Green 2006: 699-701; Gonzálvez-García 2016 [2012]: 251). 
dez resida únicamente -como defendía Hjelmslev (1980 [1943]: 28)- en su capacidad para describir el producto de la actividad lingüística. Al contrario, su objetivo parece ser el de describir la realidad de las unidades de la lengua española tal como se entiende que estas se presentan en la mente de los hablantes. Así se constata ya en los primeros trabajos de Trujillo, en los que el autor sostiene que los modelos propuestos por la lingüística deben ser el reflejo de mecanismos cognitivos reales: "No hay otros factores distintivos que los que se hallan en la conciencia (competencia) lingüística de los hablantes» (1976: 56). La bibliografía de la ESLL abunda, de hecho, en pasajes de este tipo, en los que la adecuación del modelo propuesto se argumenta por medio de afirmaciones acerca de cómo "perciben» los hablantes los significados lingüísticos (p. ej., Morera 2007: 254), o de la impresión que los usuarios de una lengua tienen acerca de las relaciones que se establecen entre los signos lingüísticos, sus significados y las «cosas» (p. ej., Trujillo 1988: 10-11).

En cuanto a los instrumentos teóricos concretos elaborados por la ESLL, el modelo planteado por Morera, en particular -como apunta Hernández Arocha (2014: 238, n. 378)-, muestra afinidades notorias con algunas de las propuestas de la lingüística cognitiva. Entre ellas, cabría destacar la similitud que se observa entre, por un lado, las «intuiciones semántico-formales», de naturaleza espacial o cuantitativa, postuladas por Morera como base de todo significado lingüístico, y, por otro, los esquemas de imagen de la lingüística cognitiva (vid. Johnson 1987; Peña Cervel 2016 [2012]). La afirmación que hace Morera acerca de que el significado lingüístico actúa siempre "como un todo unitario, y no como una síntesis de partecillas o fragmentos, de los que se puede prescindir en algún contexto como hace suponer el llamado análisis componencial puesto de moda por el estructuralismo lingüístico» (2000a: 9) recuerda igualmente al concepto de gestalt, defendido por la lingüística cognitiva como uno de los rasgos clave del lenguaje (vid. Osmańska-Lipka $2012)^{14}$. En uno de sus trabajos más recientes, Morera (2014) llega incluso a plantear la consideración del "punto de vista» como rasgo semántico de los signos lingüísticos, propuesta esta que, más que limitarse a la mera suma de una nueva «figura de contenido" a la relación anteriormente presentada (vid. Morera 2007: 5-6), apunta hacia la asunción de la relevancia que para el análisis del significado lingüístico tiene el modelo del escenario, señalado por Langacker (2008: 356-357) como uno de los arquetipos conceptuales básicos.

${ }_{14}$ También es posible encontrar propuestas -heterodoxas- de análisis componencial del significado dentro del marco de la lingüística cognitiva (Evans 2015; 2016), con la diferencia, en todo caso, de que la ESLL propone un núcleo de figuras de contenido que permanecerían constantes en todos los usos de cada una de las unidades lingüísticas, mientras que, para la lingüística cognitiva, tal condición no es necesaria. Por otro lado, hay que aclarar que Morera plantea el recurso al análisis componencial como una licencia del investigador, una aproximación al verdadero significado de las unidades lingüísticas, que es, para esta corriente, como se ha señalado, de carácter inefable. 


\section{CONCLUSIÓN}

A lo largo de este artículo he pretendido poner de relieve las dificultades con las que se enfrenta la perspectiva inmanentista a la hora de abordar el estudio del fenómeno del lenguaje en su conjunto. Como alternativa, Rosso Carrasco (2017) sugiere la posibilidad de retomar la idea coseriana de una «lingüística integral» (vid. también Kabatek 2003), basada en la coexistencia de enfoques diversos, cada uno de los cuales se encargaría de explicar una parcela distinta del fenómeno lingüístico. Así, al estructuralismo le correspondería el estudio del «sistema» de la lengua, desde una perspectiva inmanente, mientras que la lingüística cognitiva asumiría la investigación de aquellas «ordenaciones conceptuales de la experiencia que afectan a la producción e interpretación de enunciados» (Rosso Carrasco 2017: 1026).

En el presente trabajo, en cambio, más que la conveniencia de reservar una parte del fenómeno lingüístico a la lingüística cognitiva, lo que se sostiene es que el enfoque cognitivo, aplicado al estudio del lenguaje, no solo es capaz de integrar en sus modelos gran parte de las herramientas teóricas y los logros del estructuralismo, sino que, gracias a sus fundamentos filosóficos y metodológicos, permite también abordar las numerosas dimensiones que el inmanentismo ha descuidado históricamente y que -en opinión de Rosso Carrasco (2017) - está, de hecho, incapacitado para abarcar.

El abandono del inmanentismo como condición sine qua non de la lingüística y su sustitución por el enfoque cognitivo no solo no contribuye a difuminar su objeto de estudio -como desde las filas del estructuralismo podría temerse-, sino que, muy al contrario, ubica la disciplina en su espacio natural dentro del ámbito general de la investigación científica, recuperando los vínculos necesarios para la explicación tanto del uso como del origen (filogenético y ontogenético) de las unidades del lenguaje.

Por otro lado, los diversos instrumentos teóricos aportados por la lingüística cognitiva -especialmente, la teoría de la metáfora y la metonimia conceptual (Lakoff y Johnson 1986 [1980], 1999; Barcelona 2016 [2012]) - permiten dar respuesta a las numerosas críticas -incluidas las formuladas por la propia ESLL- recibidas por el estructuralismo en relación con su incapacidad para ir más allá del nivel de la denotación en la descripción semántica de las unidades lingüísticas. En este sentido, los aportes de la lingüística cognitiva pueden verse como desarrollo de lo que en la obra de la ESLL queda, por lo general, simplemente señalado, de manera general y vaga, o insuficientemente explicado por el modelo teórico propuesto.

La ESLL representa, ciertamente, un caso sumamente particular dentro del panorama del estructuralismo europeo, no solo por su cuestionamiento de principios fundamentales de la corriente en la que se inscribe, sino también por su progresivo acercamiento a los intereses y propuestas del enfoque cognitivo. Aun así, y pese a la amplitud de la perspectiva adoptada, los autores de esta escuela han venido construyendo su idea del significado lingüístico sin más instrumentos metodológicos que los aportados por el proyecto inmanente (vid. García Padrón 1997: 125-137), guiados por su intuición y sus impresiones acerca de la percepción que los hablantes tienen de su propio conocimiento lingüístico. No parece razonable que una escuela 
lingüística que basa gran parte de su labor teórica y práctica en determinados postulados acerca de la naturaleza de productos cognitivos como los conceptos o los propios significados lingüísticos continúe sin establecer al menos un diálogo con la ciencia cognitiva, cuyo objeto de estudio -teórico y empírico- son precisamente aquellos fenómenos sobre los cuales la ESLL da en la mayoría de los casos sus propias hipótesis por sentadas.

Una reformulación, en el sentido aquí señalado, de los fundamentos epistemológicos y metodológicos que sostienen la labor de la ESLL no tiene por qué conllevar la asunción acrítica de determinados modelos -como la teoría del prototipo-, cuestionados tanto por el estructuralismo como por algunos sectores dentro de la propia corriente cognitiva; ni tampoco la renuncia a algunos de los principios centrales de la Escuela, tales como el concepto de valor o la postulación de invariantes de contenido para las unidades lingüísticas. Pero sí que plantearía la necesidad de encontrar evidencias que den sustento a dichos principios, hasta ahora asumidos de forma apriorística. Sin duda, tal exigencia, más que constituir un perjuicio para la ESLL, no haría sino aportar mayor solidez a una trayectoria cuyo valor no debe ser en absoluto menospreciado. 


\section{BIBLIOGRAFÍA}

Arrivé, Michel (2014): «¿Qué sucede con la inmanencia en la reflexión lingüística y semiológica de Saussure?», Tópicos del Seminario 31: 49-69.

BarCelona, Antonio (2016 [2012]): «La metonimia conceptual», en Iraide IbarRetXe-AntuÑano y Javier Valenzuela (dirs.), Lingüistica cognitiva, Barcelona: Anthropos Editorial, 123-146.

Barsalou, Lawrence W. (1999): «Perceptual symbol systems», Behavioral and Brain Sciences 22 (4): 577-660.

Batista Rodríguez, José Juan (1997): "Trujillo, lingüista», en Manuel Almeida y Josefa Dorta (eds.), Contribuciones al estudio de la lingüistica hispánica: homenaje al profesor Ramón Trujillo, I, Barcelona: Montesinos, 21-32.

Bergen, Benjamin K. (2012): Louder than words: the new science of how the mind makes meaning, Nueva York: Basic Books.

Bondron, Jean-François (2014): «La inmanencia radical», Tópicos del Seminario 32: 15-37.

Boogatart, Ronny y Alex Reuneker (2017): «Intersubjectivity and grammar», en Barbara Dancygier (ed.) Cambridge handbook of cognitive linguistics, Cambridge: Cambridge University Press, 188205. URL: https://doi.org/10.1017/9781316339732.013; 19/09/2018.

Cifuentes Honrubia, José Luis (1992): «Teoría de los prototipos y funcionalidad semántica», Estudios de Lingüistica. Universidad de Alicante 8, 133-177.

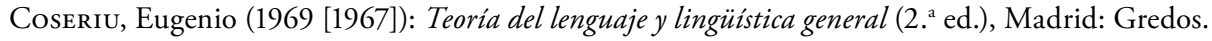

Coseriu, Eugenio (1986 [1973]): Lecciones de lingüistica general, Madrid: Gredos.

Coseriu, Eugenio (1986 [1977]): Principios de semántica estructural (2. ${ }^{a}$ ed.), Madrid: Gredos.

Coseriu, Eugenio (1990): «Semántica estructural y semántica “cognitiva”, en Jornadas de Filología. Homenaje al Prof. F. Marsá, Universidad de Barcelona, 239-282.

Dirven, René y Francisco José Ruiz de Mendoza IbáÑez (2010): «Looking back at 30 years of Cognitive Linguistics», en Elżbieta TAвaKowska, Michał Choiński y Łukasz Wiraszka (eds.), Cognitive linguistics in action: from theory to application and back, Berlín / Nueva York: Mouton de Gruyter, 13-70.

ElfFers, Els (2012): «Saussurean structuralism and cognitive linguistics», Histoire Épistémologie Langage 34 (1): 19-40.

Evans, Vyvyan (2009): How words mean: lexical concepts, cognitive models, and meaning construction, Oxford: Oxford University Press.

Evans, Vyvyan (2011): «Language and cognition: the view from Cognitive Linguistics», en Vivian Cook y Benedetta Bassetti (eds.), Language and bilingual cognition, Nueva York / Hove: Psychology Press, 69-108.

Evans, Vyvyan (2014): "Cognitive linguistics», en Susan E.F. Chipman (ed.), Oxford handbook of cognitive science, Oxford: Oxford University Press, 283-299.

Evans, Vyvyan (2015): "What's in a concept? Analog versus parametric concepts in LCCM theory», en Eric Margolis y Stephen Laurence (eds.), The conceptual mind: new directions in the study of concepts, Cambridge (Massachusetts): MIT Press, 251-290.

Evans, Vyvyan (2016): «Design features for linguistically-mediated meaning construction: the relative roles of the linguistic and conceptual systems in subserving the ideational func- 
tion of language», Frontiers in Psychology 7 (156): 1-12. URL: https://doi.org/10.3389/ fpsyg.2016.00156; 19/09/2018.

Evans, Vyvyan y Melanie Green (2006): Cognitive linguistics: an introduction, Edimburgo: Edinburg University Press.

Figueroa, Esther (1994): Sociolinguistic metatheory, Oxford: Pergamon.

FAUCONNIER, Gilles (1999): «Creativity, simulation, and conceptualization», comentario a Lawrence W. Barsalou (1999), Behavioral and Brain Sciences 22 (4): 615.

García Padrón, Dolores (1989): «Nuevas consideraciones sobre los aspectos significativo y denotativo de ciertos signos", Estudios Filológicos 24: 5-10.

García Padrón, Dolores (1990): «Alcance y límites de la investigación de los sistemas semánticos», en Actas del Congreso de la Sociedad Española de Lingüistica. XX Aniversario, I, Madrid: Gredos, 476-484.

García Padrón, Dolores (1997): El estudio semántico del lenguaje, Santa Cruz de Tenerife: Dirección General de Universidades e Investigación.

Geeraerts, Dirk (1985): Paradigm and paradox: explorations into a paradigmatic theory of meaning and its epistemological background, Lovaina: Leuven University Press.

Geeraerts, Dirk (1993): «Cognitive semantics and the history of philosophical epistemology», en Richard A. Geiger y Brygida Rudzka-Ostyn (eds.), Conceptualizations and mental processing in language. A selection of papers from the First International Cognitive Linguistics Conference in Duisburg, 1989, Berlín/Nueva York: Mouton de Gruyter, 163-189.

Geeraerts, Dirk (2016 [1989]): «Prospects and problems of prototype theory», Diacronia 3 (A53): $1-16$.

Gibss, Raymond W. Jr. (1996): «Why many concepts are metaphorical», Cognition 61: 309-319. URL: https://doi.org/10.1016/S0010-0277(96)00723-8; 19/09/2018.

Gibbs, Raymond W. Jr. (2005): Embodiment and cognitive science, Nueva York: Cambridge University Press.

Goldberg, Adele (2006): Constructions at work: the nature of generalization in language, Nueva York: Oxford University Press.

Goldstone, Robert L., Alan Kersten y Paulo F. Carvalho (2017): "Categorization and concepts», en J. Wixted (ed.) Stevens' handbook of experimental psychology and cognitive neuroscience (4. ${ }^{\mathrm{a}}$ ed.), vol. 3: Language and thought, Nueva Jersey: Wiley, 275-317.

GonZÁLVEZ-García, Francisco (2016 [2012]): «La(s) gramática(s) de construcciones», en Iraide IBArRetXe-Antuñano y Javier Valenzuela (dirs.), Lingüistica cognitiva, Barcelona: Anthropos Editorial, 249-280.

Gries, Stefan Th. (2006): "Corpus-based methods and cognitive semantics: the many senses of to run", en Stefan Th. Gries y Anatol Stefanowitsch (eds.), Corpora in cognitive linguistics: corpus-based approaches to syntax and lexis, Berlín: Mouton de Gruyter, 57-99.

Harder, Peter (2009): "Meaning as input: the instructional perspective», en Vyvyan Evans y Stéphanie Pourcel (eds.), New directions in cognitive linguistics, Ámsterdam: John Benjamins, 15-26. URL: https://doi.org/10.1075/hcp.24.05har; 10/11/2018.

HASER, Verena (2005): Metaphor, metonymy, and experientialist philosophy: challenging cognitive semantics, Berlín / Nueva York: Mouton de Gruyter. 
Hernández Arocha, Héctor (2014): Las familias de palabras. Relaciones entre morfología, semántica y estructura argumental en las raices «dec(ir)» $y$ «sag(en)», Berlín/Boston: De Gruyter.

Hessen, Johannes (1970 [1926]): Teoría del conocimiento (12. ${ }^{\text {e }}$ ed.), Madrid: Espasa-Calpe.

HJelmslev, Louis (1980 [1943]): Prolegómenos a una teoría del lenguaje, Madrid: Gredos.

JoHnson, Mark (1987): The body in the mind: the bodily basis of meaning, reason, and imagination, Chicago: Chicago University Press.

Johnson, Mark y George Lakoff (2002): «Why cognitive linguistics requires embodied realism», Cognitive Linguistics 13 (3): 245-263.

Каватек, Johannes (2003): «Unidad de significado, designado y lingüistica integral», Odisea 3: 87-100.

Kemmer, Suzanne y Michael BArLow (2000): «Introduction: a usage-based conception of language», en Michael Barlow y Suzanne Kemmer (eds.), Usage-based models of language, Stanford: CSLI Publications, VII-XxvIII.

Kleiber, Georges (1995 [1990]): La semántica de los prototipos, Madrid: Visor Libros.

Komatsu, Lloyd (1992): «Recent views of conceptual structure», Psychological Bulletin 112 (3): 500526. URL: https://doi.org/10.1037/0033-2909.112.3.500; 19/09/2018.

LAKOFF, George (1987): Women, fire and dangerous things: what categories reveal about the mind, Chicago: Chicago University Press.

LAKOFF, George (1990): «The invariance hypothesis: is abstract reason based on image-schemas?», Cognitive Linguistics 1 (1): 39-74. URL: https://doi.org/10.1515/cogl.1990.1.1.39; 10/11/2018.

Lakoff, George y Mark Johnson (1986 [1980]): Metáforas de la vida cotidiana, Madrid: Ediciones Cátedra.

Lakoff, George y Mark Johnson (1999): Philosophy in the flesh: the embodied mind and its challenge to Western thought, Nueva York: Basic Books.

LANGacker, Ronald W. (1987): Foundations of cognitive grammar, vol. 1, Stanford: Stanford University Press.

Langacker, Ronald W. (2007): «Cognitive grammar», en Dirk Geeraerts y Hubert Cuyckens (eds.), The Oxford handbook of cognitive linguistics, Oxford: Oxford University Press, 421462 .

LANGaCKer, Ronald W. (2008): Cognitive grammar: a basic introduction, Nueva York: Oxford University Press.

LANGACKer, Ronald W. (2009): «A dynamic view of usage and language acquisition», Cognitive Linguistics 20 (3): 627-640.

Lyons, John (1980): Semántica, Barcelona: Teide.

Morera, Marcial (2000a): Apuntes para una gramática del español de base semántica, vol. I: Morfología, Puerto del Rosario: Servicio de Publicaciones del Cabildo de Fuerteventura.

Morera, Marcial (2000b): Apuntes para una gramática del español de base semántica, vol. II: Sintaxis, Puerto del Rosario: Servicio de Publicaciones del Cabildo de Fuerteventura.

Morera, Marcial (2005): La complementación morfológica en español. Ensayo de interpretación semántica, Frankfurt am Main: Peter Lang.

Morera, Marcial (2007): La gramática del léxico español, Badajoz: Abecedario. 
Morera, Marcial (2014): «El punto de vista del observador como rasgo semántico de los signos descriptivos», Verba 41: 75-102.

Nuyts, Jan (2007): «Cognitive linguistics and functional linguistics», en Dirk Geeraerts y Hubert Cuyckens (eds.), The Oxford handbook of cognitive linguistics, Oxford: Oxford University Press, 543-565.

OsmańsKa-Lipka, Iwona (2012): «Elements of Gestalt psychology in American cognitive linguistics», Annales Universitatis Mariae Curie-Sktodowska 30 (2): 47-72.

Osuna García, Francisco (2005): «¿Es posible una lingüística inmanente?», Language Design 7: 51-84.

PAOLuCCI, Claudio (2014): «El principio de inmanencia como fundamento de la epistemología semiótica», Tópicos del Seminario 32: 93-122.

Peña Cervel, M. a Sandra (2016 [2012]): «Los esquemas de imagen», en Iraide Ibarretxe-Antuñano y Javier Valenzuela (dirs.), Lingüistica cognitiva, Barcelona: Anthropos Editorial, 69-96.

Real Academia española (2014): Diccionario de la lengua española (DRAE) (23. ${ }^{a}$ ed.), Madrid: Espasa. URL: http://www.rae.es/; 10/11/2018.

Radden, Günter y René Dirven (2007): Cognitive English grammar, Ámsterdam/Filadelfia: John Benjamins.

RAKova, Marina (2002): «The philosophy of embodied realism: a high price to pay?», Cognitive Linguistics 13 (3): 215-244.

Rosch, Eleanor (1999 [1978]): «Principles of categorization», en Eleanor Rosch y Barbara B. LLOyD (eds.), Cognition and categorization, Hillsdale, Nueva Jersey: Lawrence Erlbaum, 27-48.

Rosso Carrasco, Miguel (2017): «¿Es consistente una semántica del hablar desde los presupuestos teóricos del estructuralismo?», Rilce. Revista de Filología Hispánica 33 (3): 992-1031. URL: https://doi.org/10.15581/008.33.3.992-1031; 19/09/2018.

Ruiz de Mendoza Ibáñez, Francisco y Alicia Galera Masegosa (2014): Cognitive modeling: a linguistic perspective, Ámsterdam: John Benjamins.

Saussure, Ferdinand de (1991 [1916]): Curso de lingüistica general, Madrid: Alianza Editorial.

Sото, Guillermo (2001): «Perspectivas para la lingüística: más allá de la dicotomía formalismo/funcionalismo", Revista Chilena de Humanidades 21: 115-154.

TAYLOR, John R. (1999): «Cognitive semantics and structural semantics», en Andreas Blank y Peter Косн, Historical semantics and cognition, Berlín/Nueva York: Mouton de Gruyter, 17-48.

TAYLOR, John R. (2003): Linguistic categorization (3. ${ }^{a}$ ed.), Oxford: Oxford University Press.

Tomasello, Michael (2013 [2008]): Los orígenes de la comunicación humana, Madrid: Katz Editores.

Trujillo, Ramón (1976): Elementos de semántica lingüistica, Madrid: Cátedra.

Trujillo, Ramón (1988): Introducción a la semántica española, Madrid: Arco Libros.

Trujillo, Ramón (1998): «Para una discusión del concepto de campo semántico», en Gerd Wotjak (coord.), Teoría del campo y semántica léxica, Frankfurt am Main: Peter Lang, 87-125.

Valenzuela, Javier, Iraide IbarretXe-Antuñano y Joseph Hilferty (2016 [2012]): «La semántica cognitiva», en Iraide Ibarretxe-Antuñano y Javier Valenzuela (dirs.), Lingüistica cognitiva, Barcelona: Anthropos Editorial, 41-68.

Wierzbicka, Anna (1996): Semantics: primes and universals, Oxford/Nueva York: Oxford University Press. 
Zahavi, Dan (2009): «Naturalized phenomenology», en Shaun Gallagher y Daniel Schmicking (eds.), Handbook of phenomenology and cognitive science, Dordrecht: Springer, 3-19.

Zinna, Alessandro (2014): «La inmanencia: línea de fuga semiótica», Tópicos del Seminario 31: 19-47.

ZInnA, Alessandro (2015): «Una mirada retrospectiva sobre la inmanencia», Tópicos del Seminario 33: 335-358.

Zlatev, Jordan (2005): «What's in a schema? Bodily mimesis and the grounding of language», en Beate Hampe (ed.), From perception to meaning: image schemas in cognitive linguistics, Berlín / Nueva York: Mouton de Gruyter, 313-343. URL: https://doi.org/10.1515/97831101 97532.4.313; 19/09/2018.

Zlatev, Jordan (2007): «Embodiment, language, and mimesis», en Tom Ziemke, Jordan Zlatev y Roslyn M. Frank (eds.), Body, language and mind, vol. 1: Embodiment, Berlín/Nueva York: Mouton de Gruyter, 297-337.

Zlatev, Jordan (2009): «Phenomenology and cognitive linguistics», en Shaun Gallagher y Dan Schmicking (eds.), Handbook of phenomenology and cognitive science, Dordrecht: Springer, 297-337. 